(2)isma

\title{
$\mathrm{JG} \mid \mathrm{U}$
}

JOHANNES GUTENBERG

UNIVERSITÄT MAINZ

\section{Upgrade of the ATLAS Level-1 Trigger with an FPGA based Topological Processor}

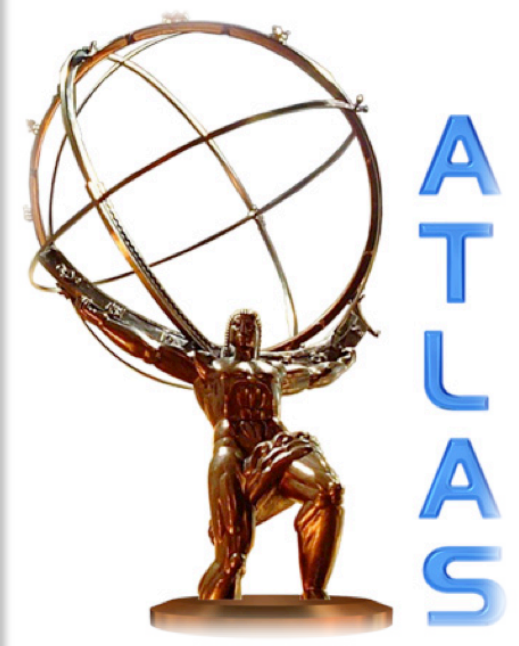

\author{
R. Caputo \\ Institute for Physics, Universität Mainz \\ on behalf of the ATLAS collaboration
}

27 October 2013

2013 IEEE-NSS Conference

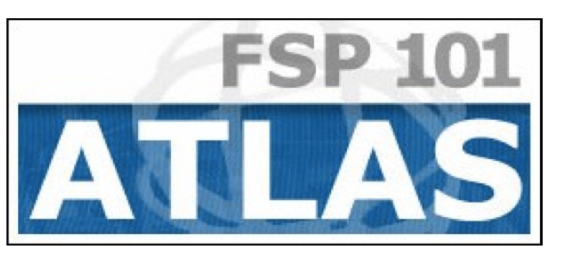




\section{Overview}

- Introduction

- The LHC and ATLAS

- Running conditions 2012/2015

- The Level-1 Trigger

- Level-1 Topological Trigger

- Prototype Specifications

- Tests and first results

- Summary 


\section{Introduction To ATLAS}

Right handed coordinate system $x$-coordinate toward $\mathrm{LHC}$ ring pseudorapidity, $\eta=\ln (\tan (\theta / 2))$

Inner Detector:

Tracking to identify charged particles
Muon Systems:

Tracking to identify /muons, magnets to bend trajectory

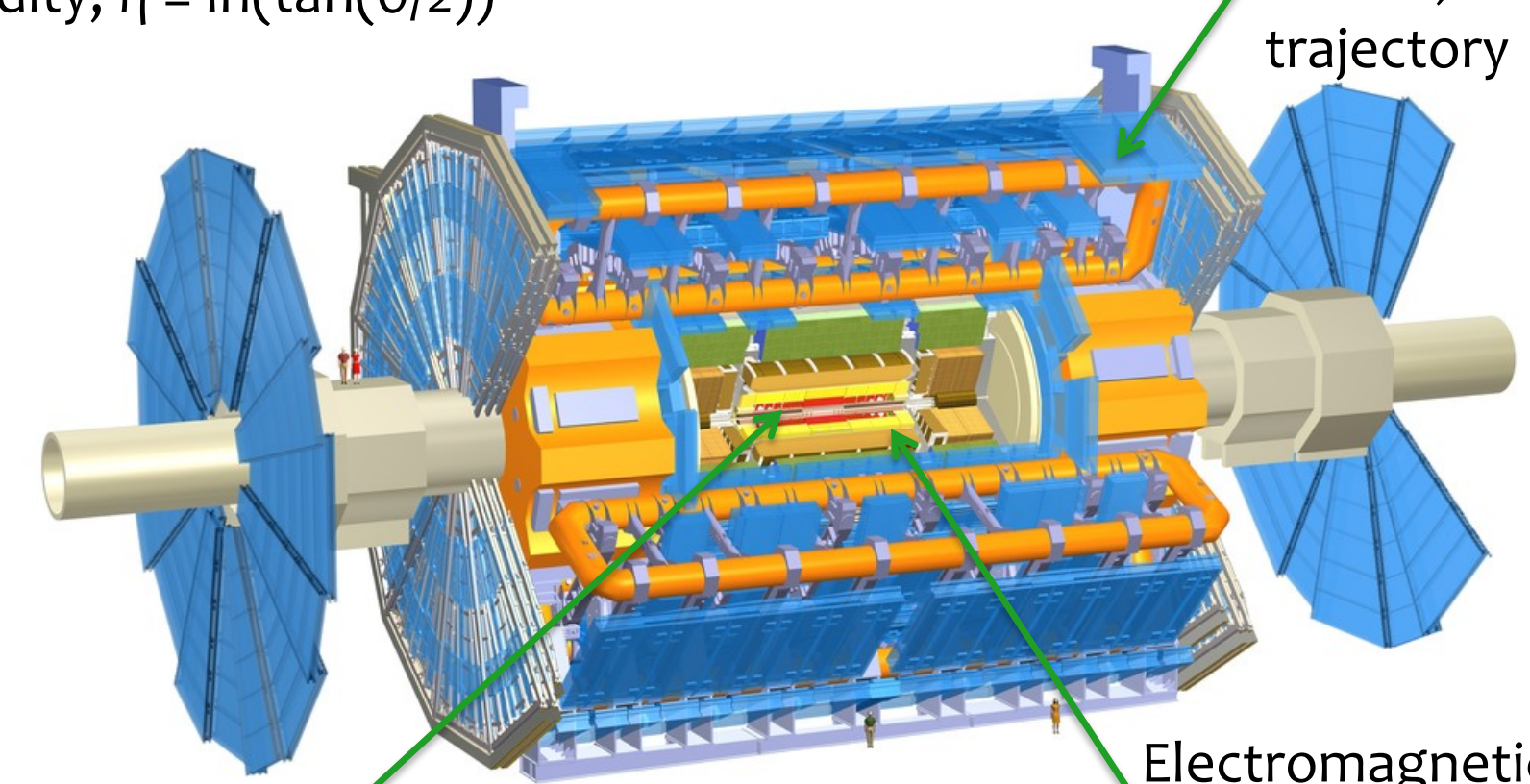

Electromagnetic and Hadronic Calorimeters: Energy deposition of EM and Hadronic particles 


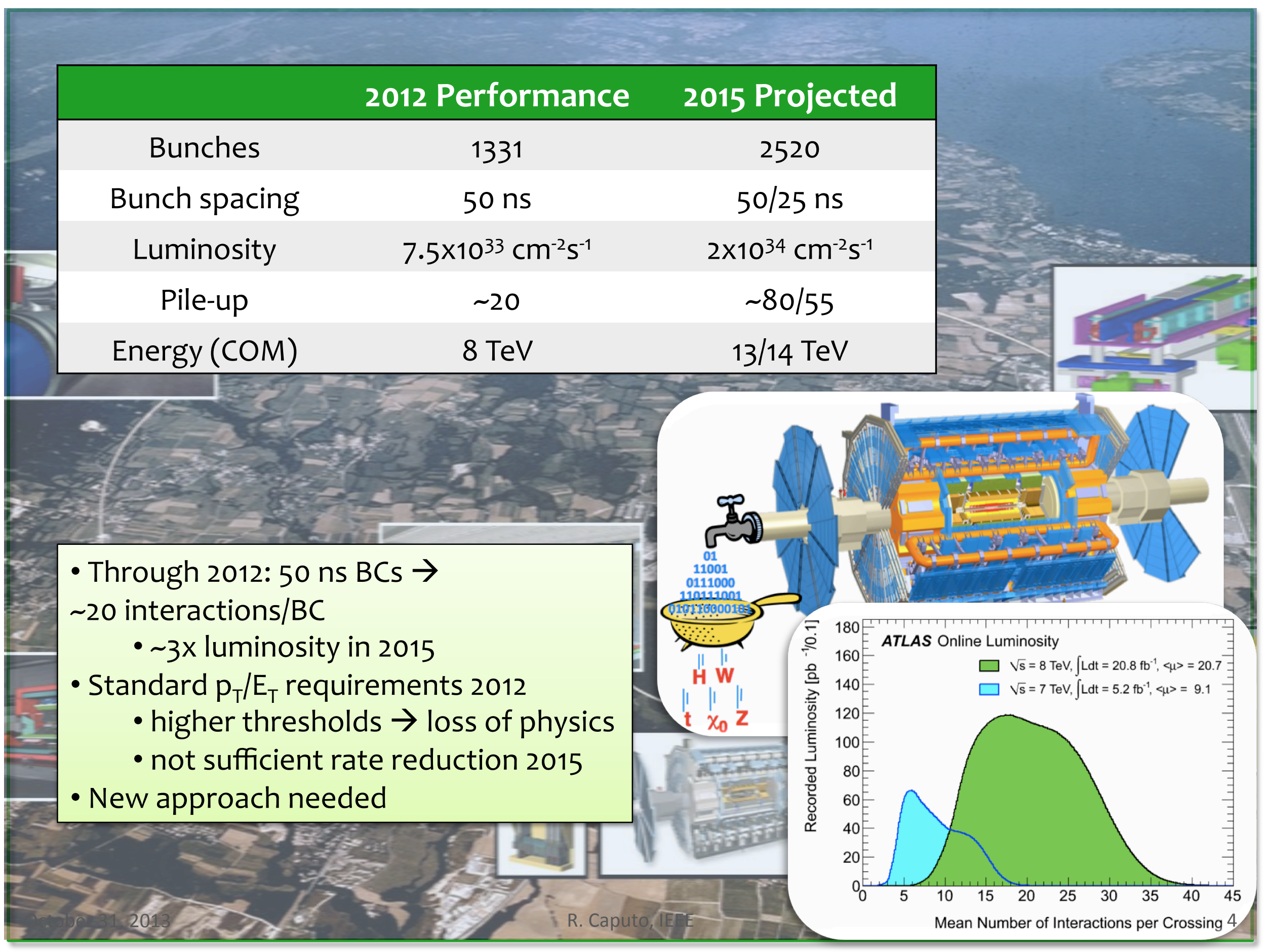




\section{Trigger Menu 2012/2015}

\begin{tabular}{cccc} 
Trigger Type & L1 Item & $\begin{array}{c}\text { Offline Threshold } \\
{[\mathrm{GeV}]}\end{array}$ & $\begin{array}{c}\text { Rate } \\
{[\mathbf{k H z}]}\end{array}$ \\
\hline $\begin{array}{c}\text { Single electron/ } \\
\text { photon }\end{array}$ & $\begin{array}{c}\text { EM30(EM18VH) } \\
\text { EM30 }\end{array}$ & $\begin{array}{c}45(25) \\
100\end{array}$ & $\begin{array}{c}7.5(20.5) \\
7.5\end{array}$ \\
\hline Single muon & MU15 & 25 & 11 \\
\hline Single tau & TAU40 & 120 & 4 \\
\hline Single jet & J75 & 165 & 2 \\
\hline $\begin{array}{c}\text { Single electron/ } \\
\text { photon }\end{array}$ & EM40H(EM28H) & $50(33)$ & $8 \mathrm{TeV}, 1 \times 10^{34} \mathrm{~cm}^{-2} \mathrm{~s}^{-1}$ \\
\hline Single muon & EM50 & 60 & $7(28)$ \\
\hline Single tau & MU20 & 25 & 26 \\
\hline Single jet & TAU60 & 150 & 10 \\
\hline J100 & 250 & $14 \mathrm{TeV}, 2 \times 10^{34} \mathrm{~cm}^{-2} \mathrm{~s}^{-1}$ \\
\hline
\end{tabular}




\section{The Level 1 Trigger}

Calorimeters

Muon Detectors

The task of Level 1:

reduce rate from

$\sim 40 \mathrm{MHz}$ to $\sim 70 \mathrm{kHz}$ in 2012

2015 L1 up to $\sim 100 \mathrm{kHz}$

How is it done:

custom built hardware information on Regions of Interest (ROIs)

Three systems: L1 Muon, L1 Calorimeter (L1Calo), Central Trigger Processor (CTP) L1 sends information to CTP which makes the decision

$$
\text { latency } \sim 2.5 \mu \mathrm{s}
$$

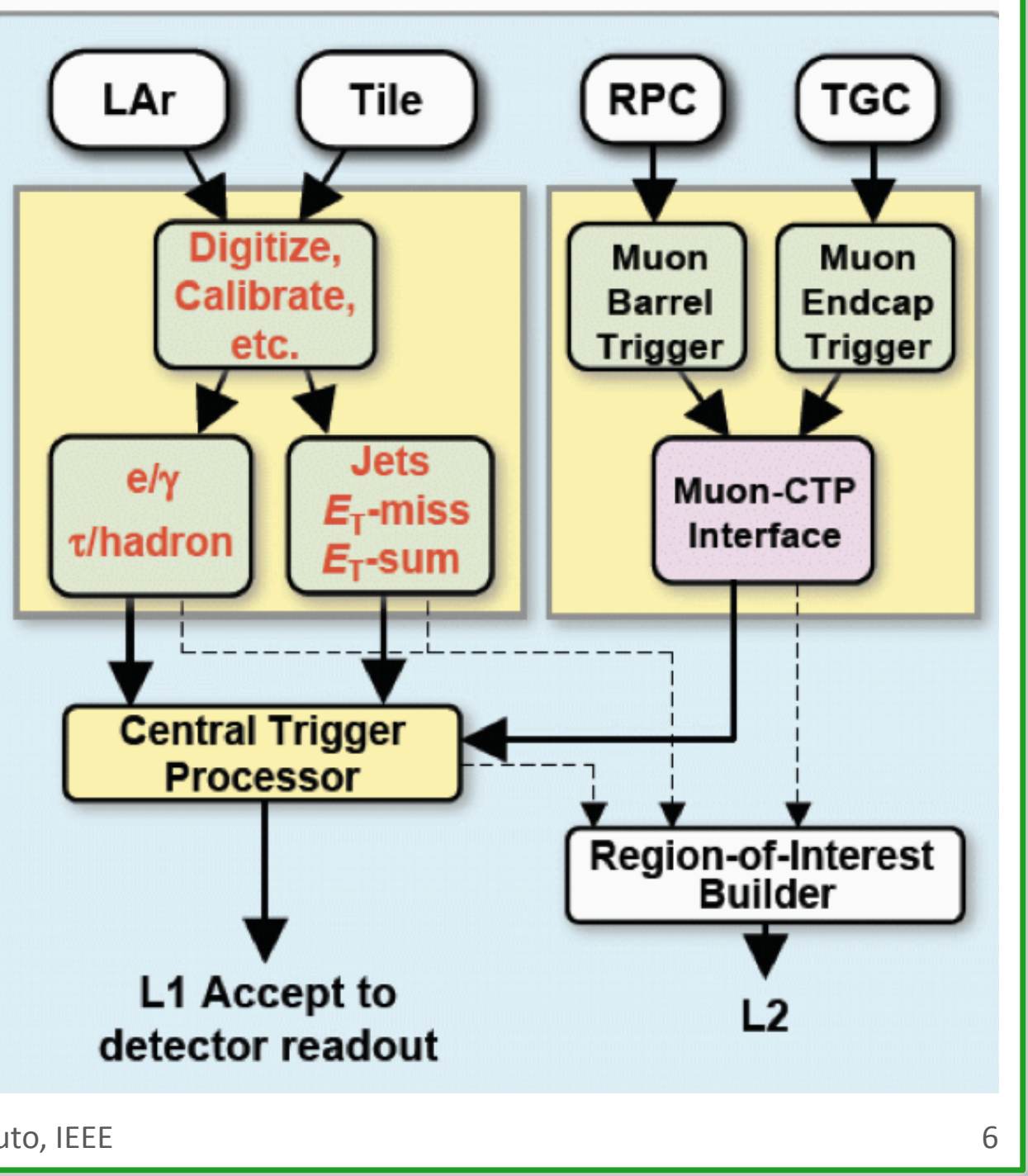




\section{L1Topo: A Solution to the Rate}

\section{What is L1Topo?}

Examples Online trigger decision based on event topology

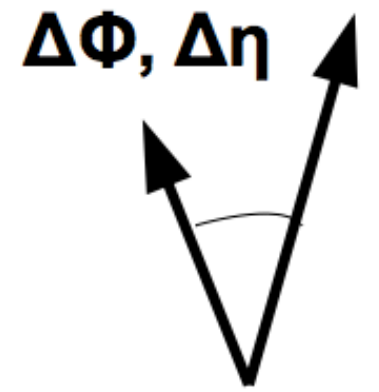

Isolation,

overlap removal,

b-tagging...

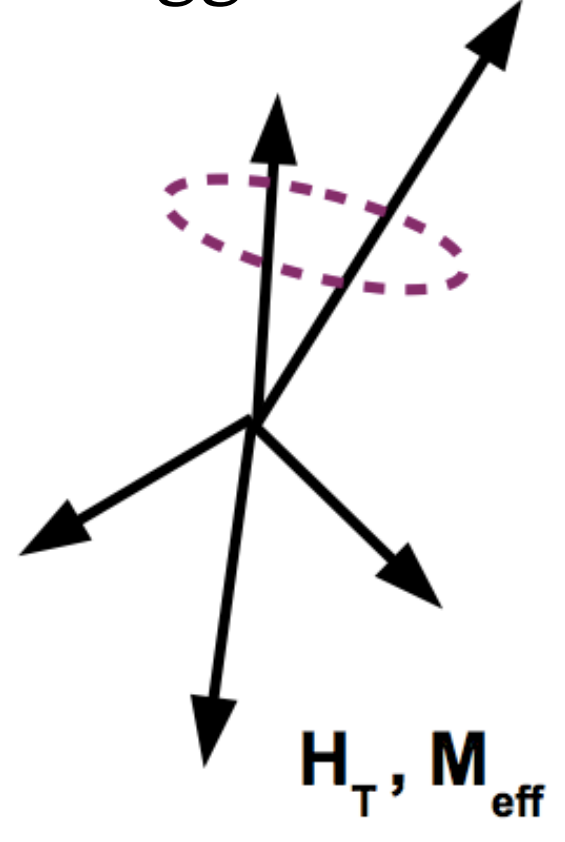

Fat jets

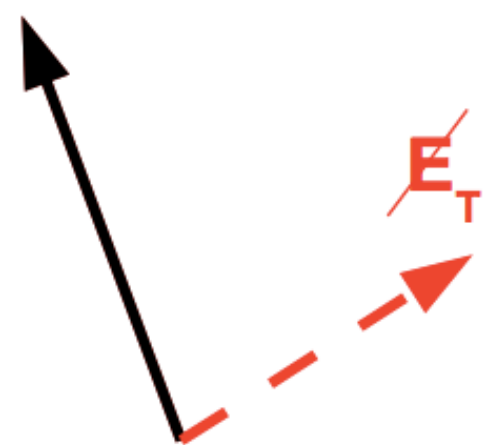

Transverse Mass, $\Delta \Phi\left(\right.$ jet, $\left.E_{T}\right)$

TOB: Trigger OBject data 


\section{A Physics Case: $\quad H \rightarrow \tau \tau$}

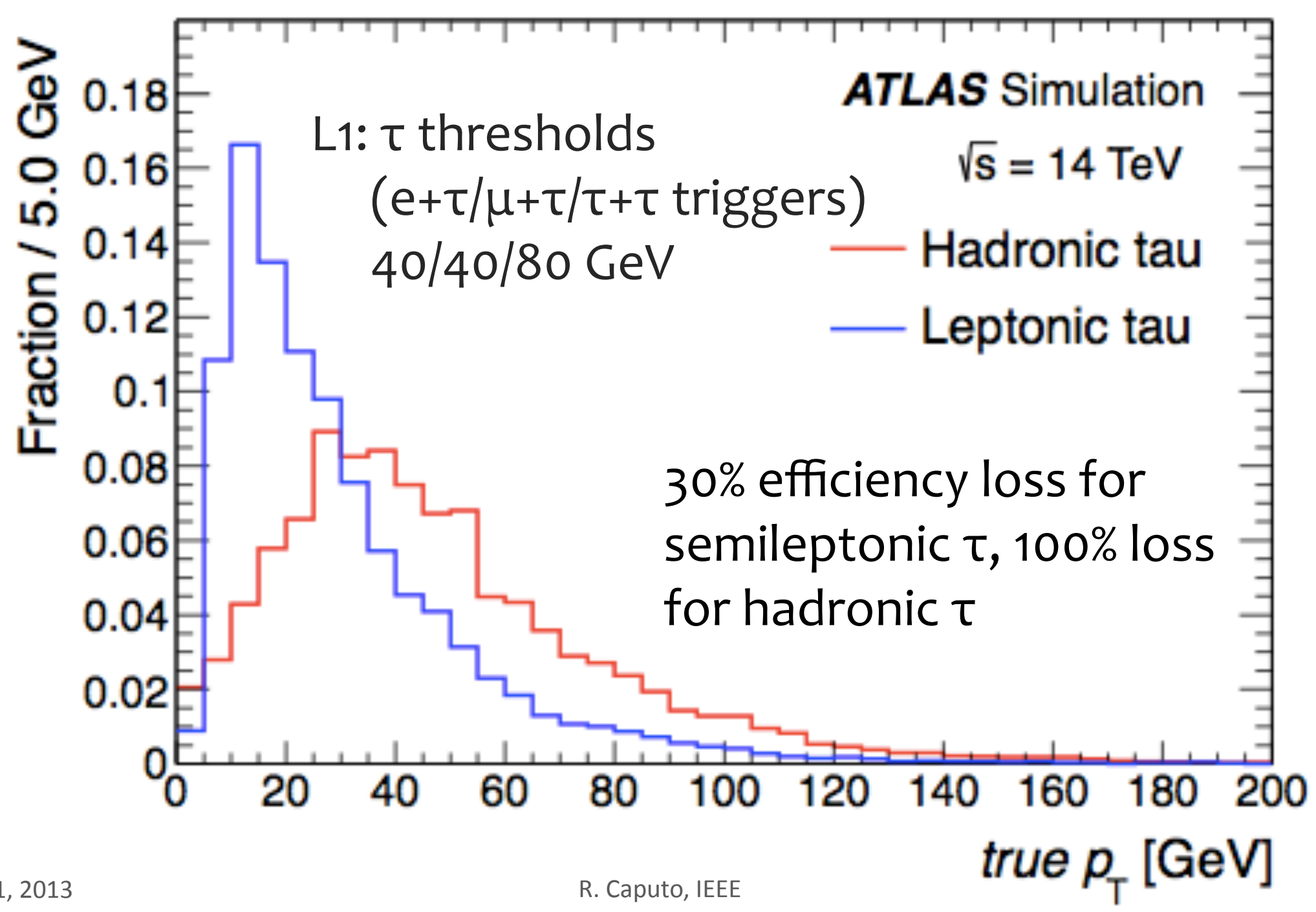




\section{A Physics Case: $\quad H \rightarrow \tau \tau$}

How can this be saved?

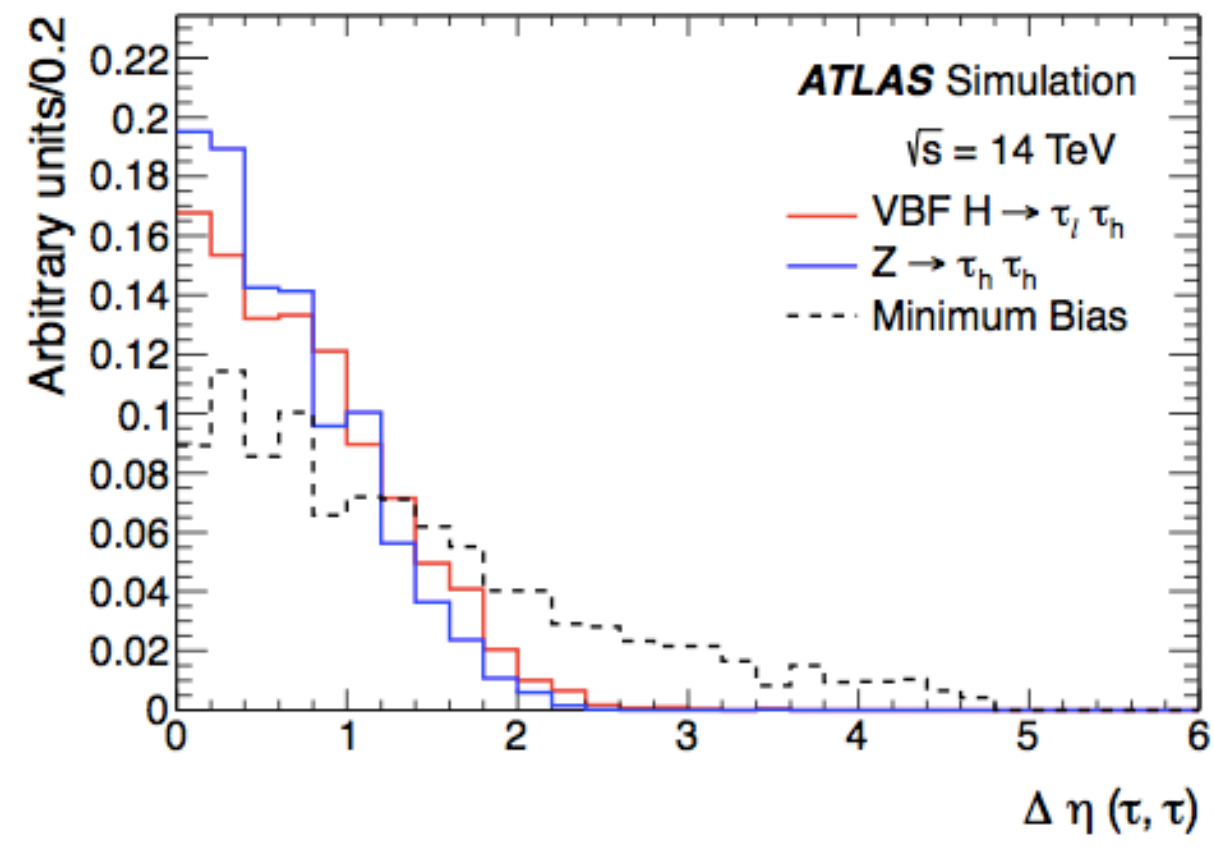

Topological Trigger!

Example 1: $\Delta \eta(\tau, \tau)<2$ and tight isolation

L1 rate reduced $\sim 2$ - preliminary

This would be enough to stabilize thresholds at current levels 


\section{L1Calo Current System}

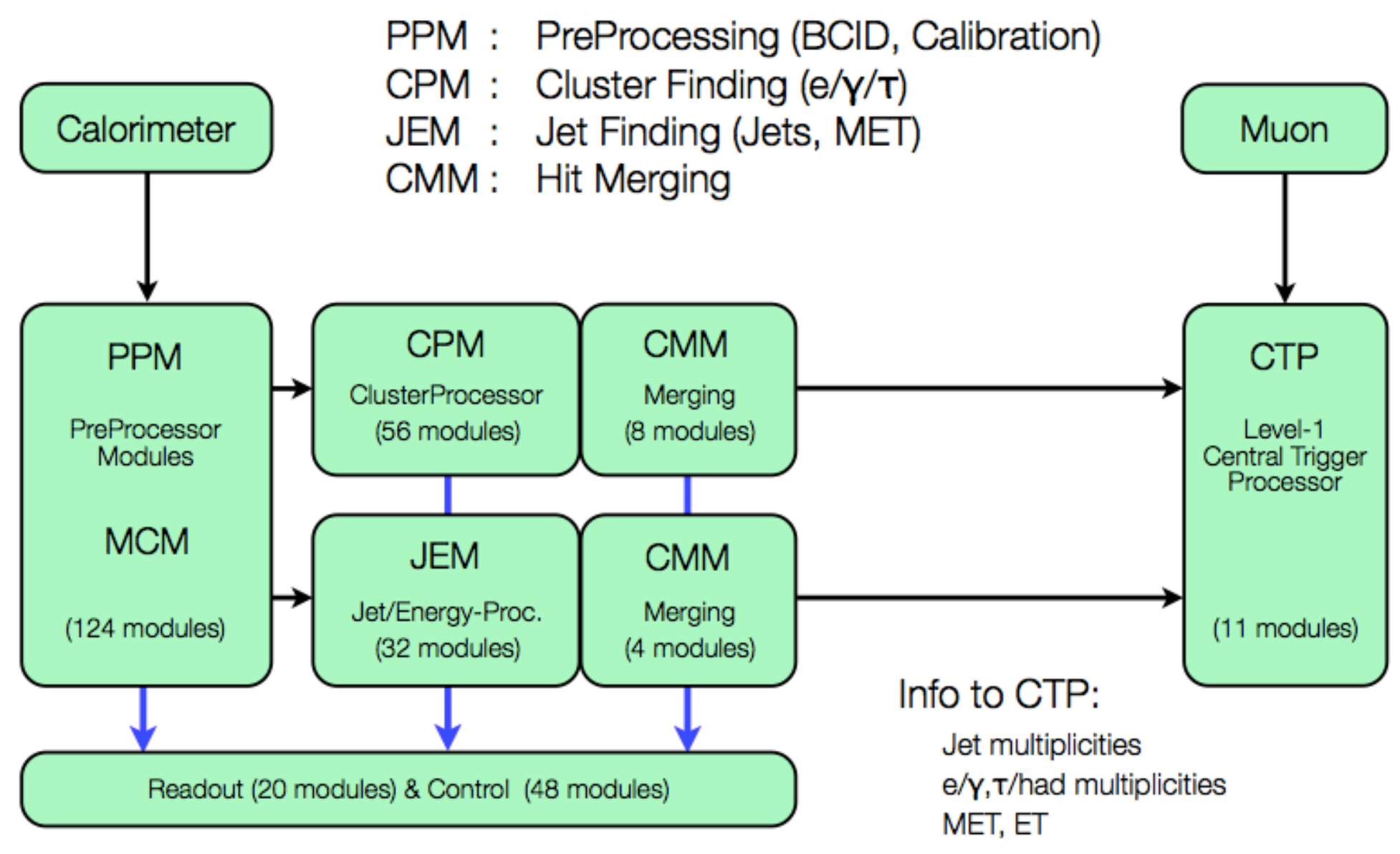




\section{L1Calo + L1 Topological Processor}

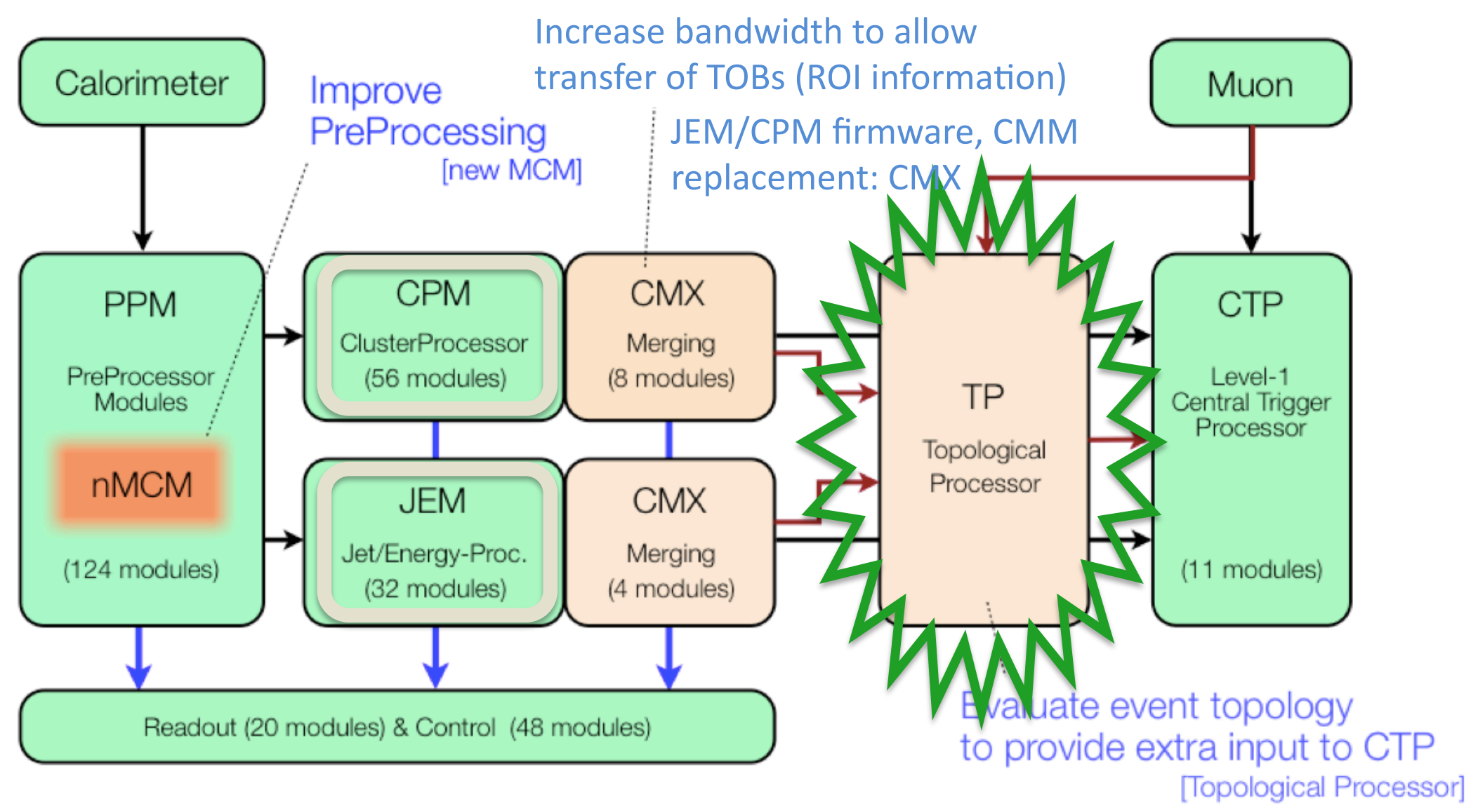

rate increase on backplane to $\mathrm{CMX}$ 


\section{L1Calo + L1 Topological Processor}

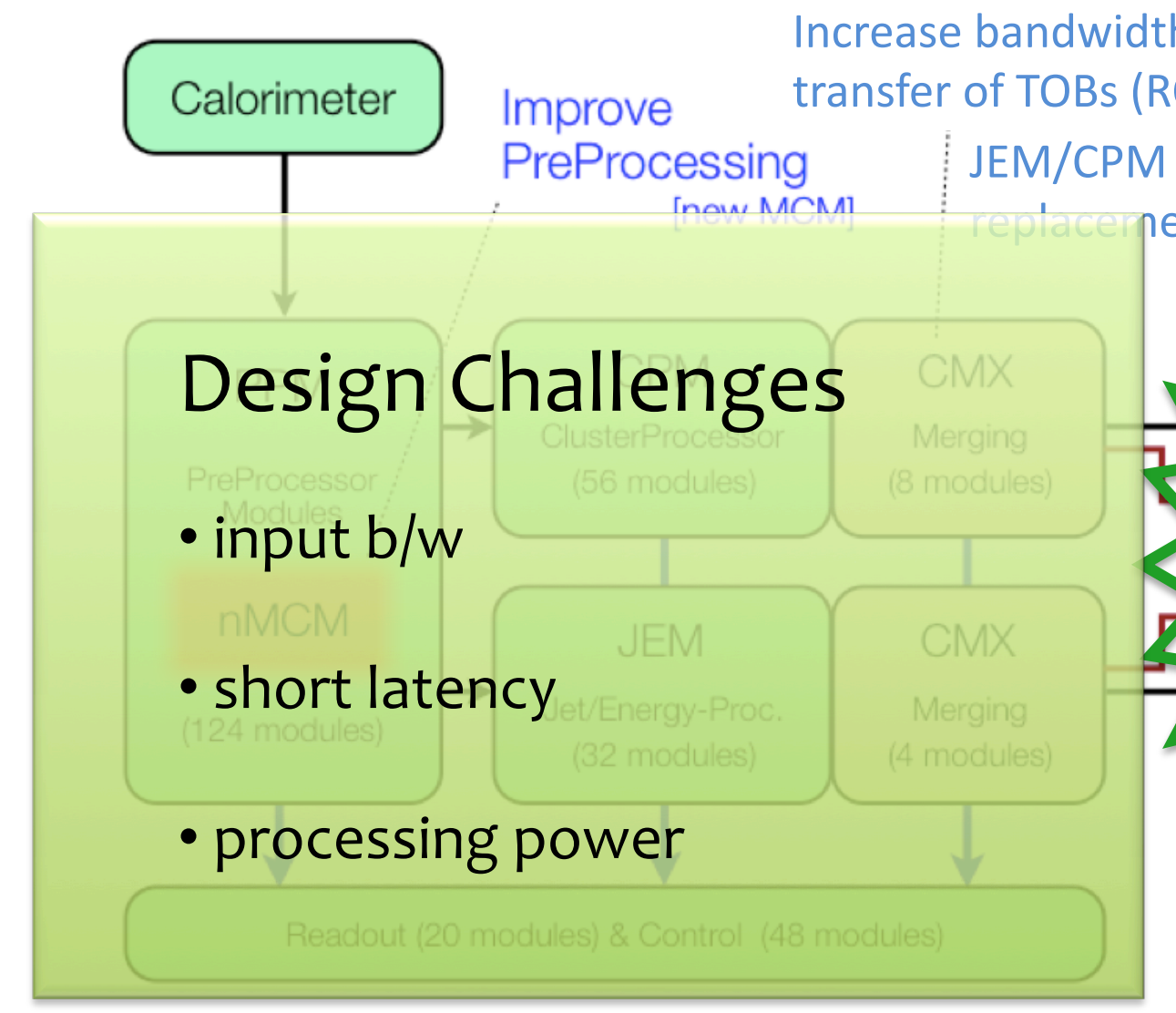

rate increase on backplane to $\mathrm{CMX}$

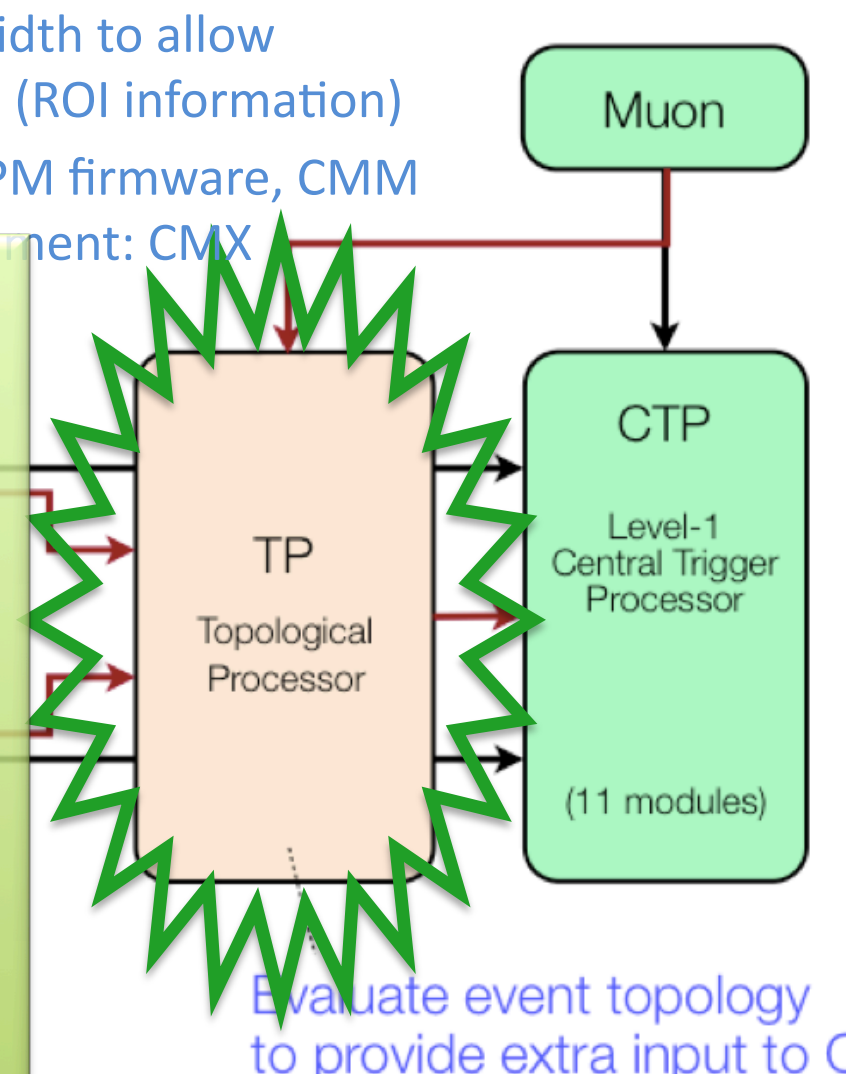

[Topological Processor] 


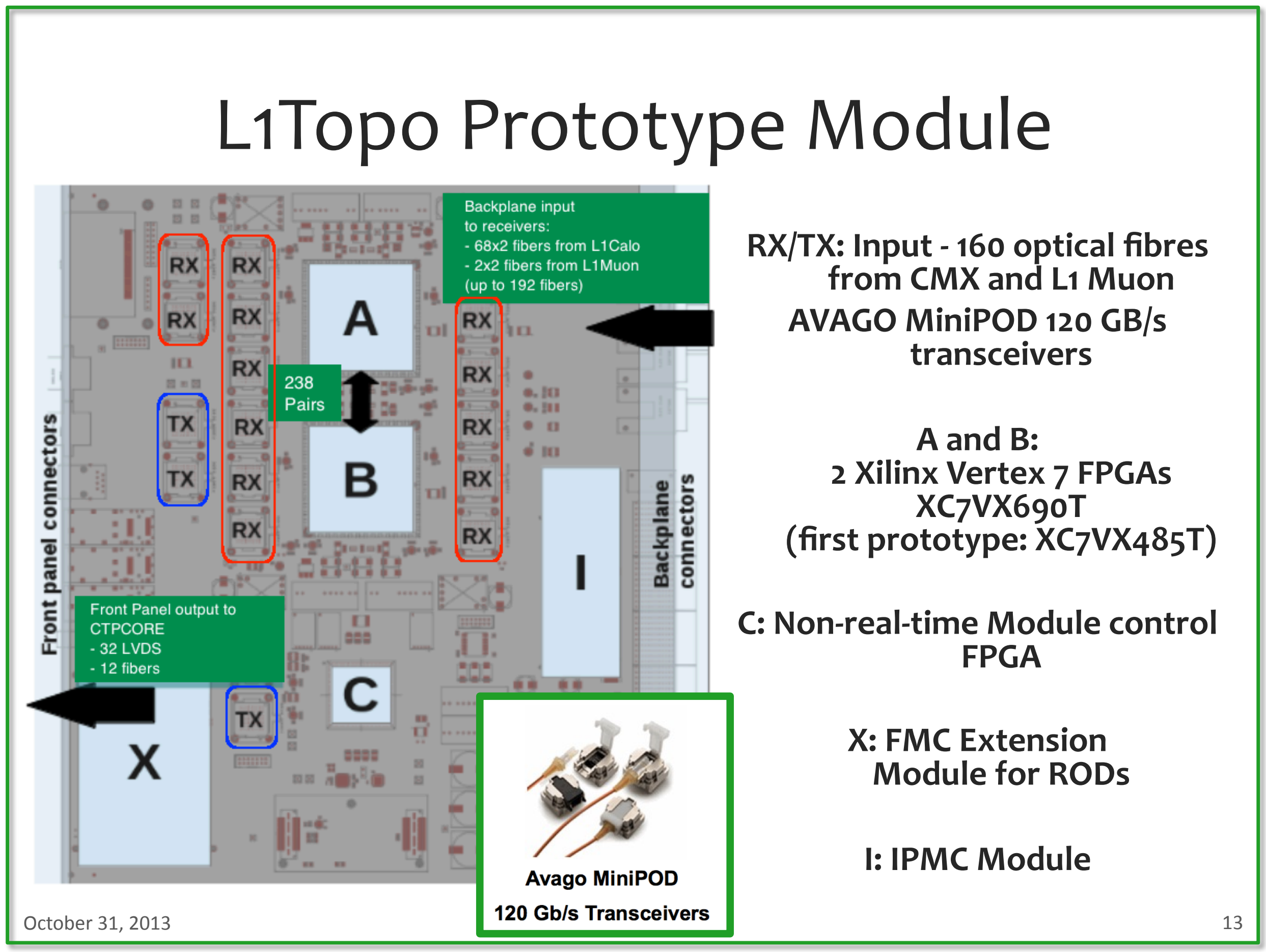




\section{L1Topo Prototype Module}

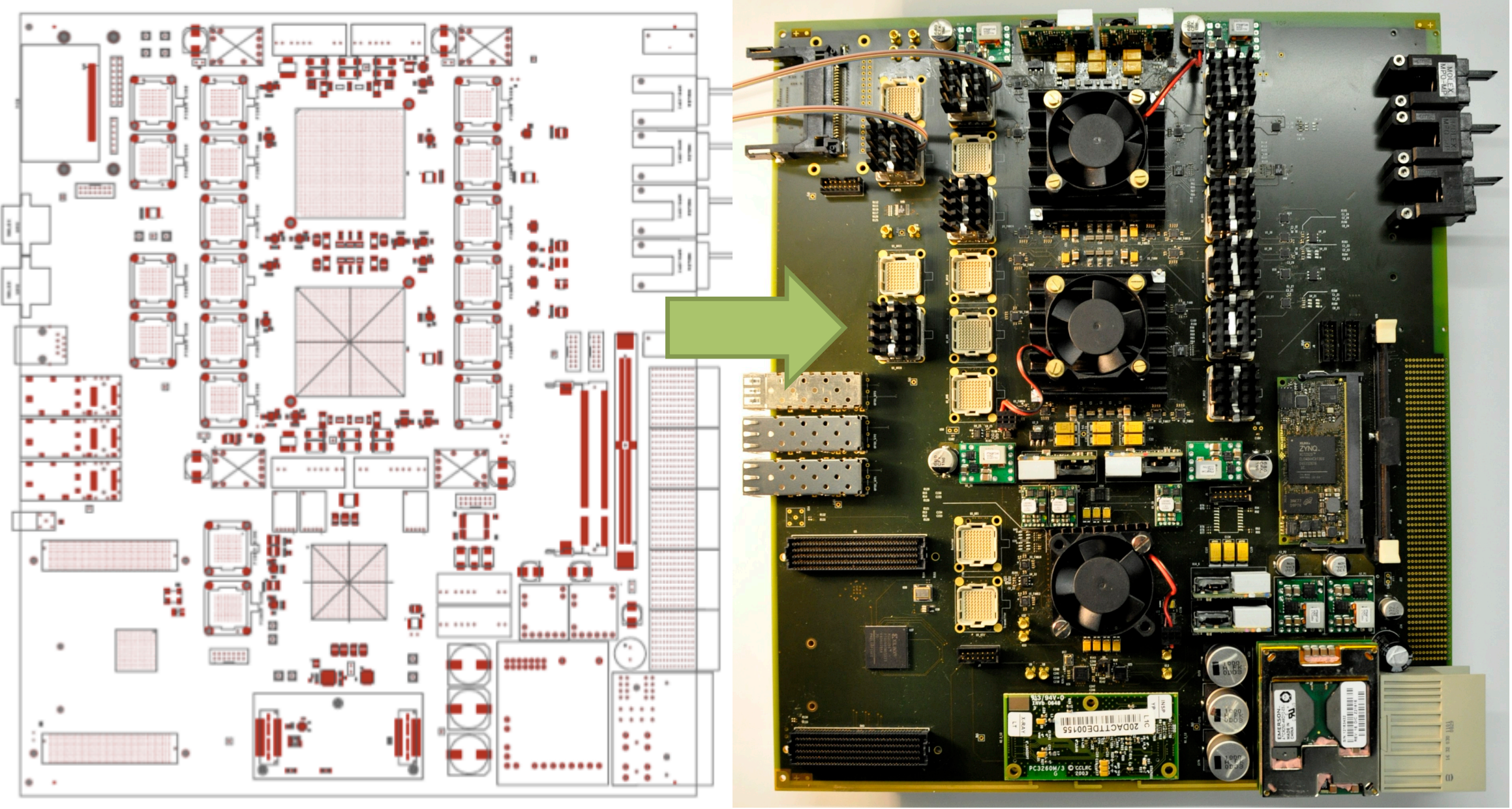




\section{L1Topo Prototype Module}

\section{Prototype Specifications}

- Fully ATCA compliant

- 18 MiniPOD sockets via 48-way backplane connectors

- Vertex 7 FPGAs

- XC7VX690T

- first prototype: XC7VX485T

- Module control via a large "Kintex" FPGA

- Output to CTP

- 12-way optical

- 32-way electrical (LVDS)

- Embedded ROD on control FPGA up to $12 \mathrm{DAQ} /$ Rol fibers

- Beyond the prototype

- larger capacity, more 10

- 56 vs. 80 links

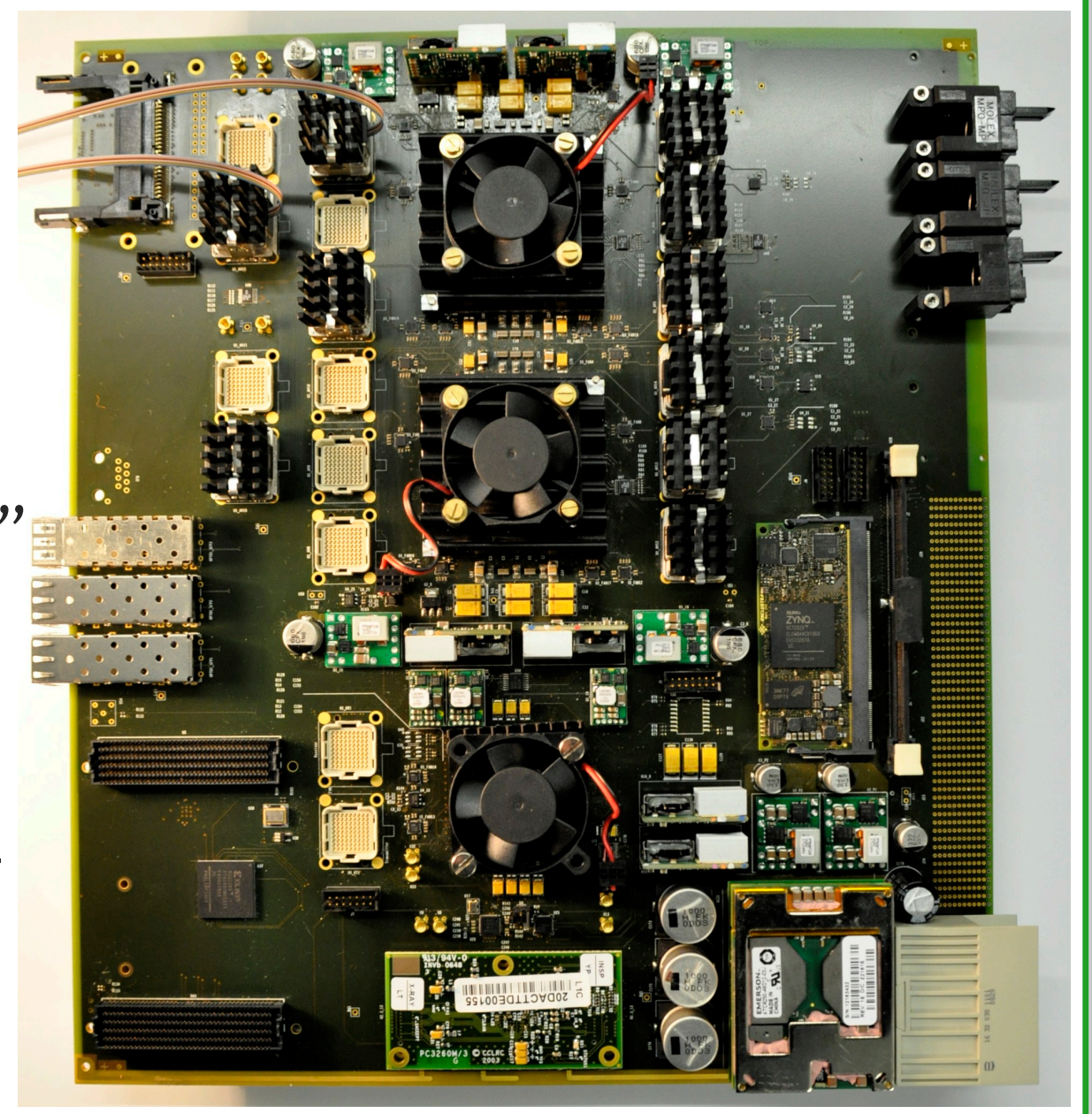




\section{High Speed Link Test}

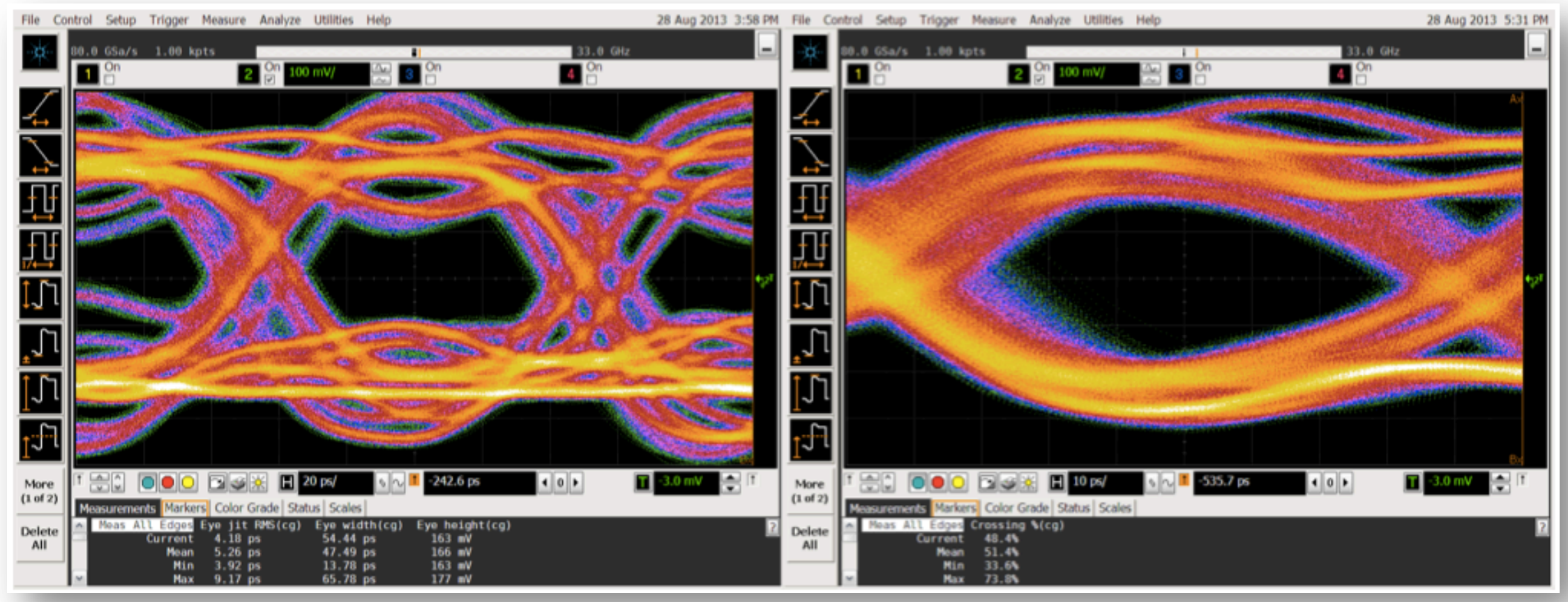

- Eye measurement @10.26 GB/s

- miniPOD RX (left), miniPOD TX (right) 


\section{Real Time High Speed Links Tests}

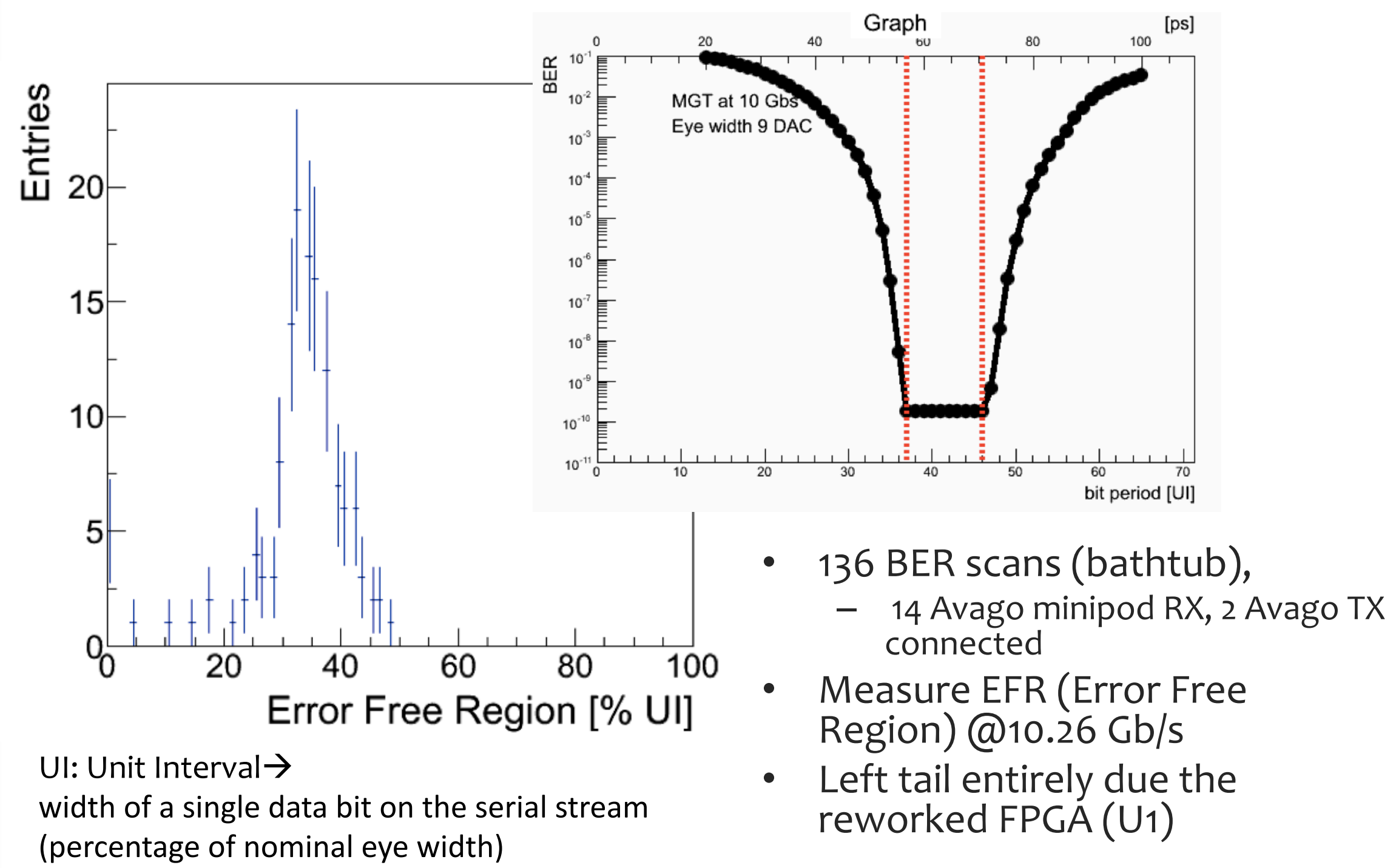




\section{Algorithm Implementation on FPGA}

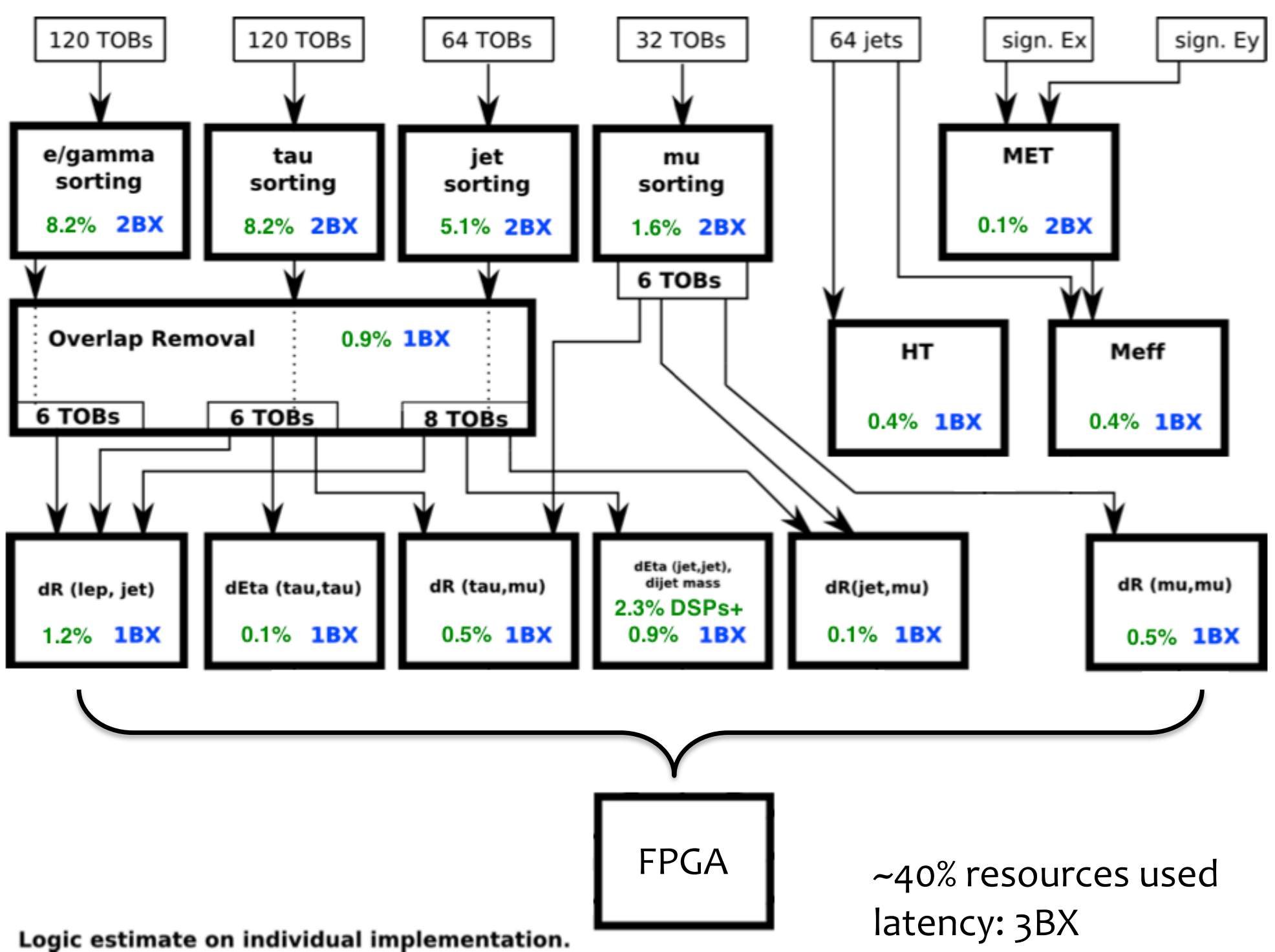




\section{Resource Usage vs TOB number}

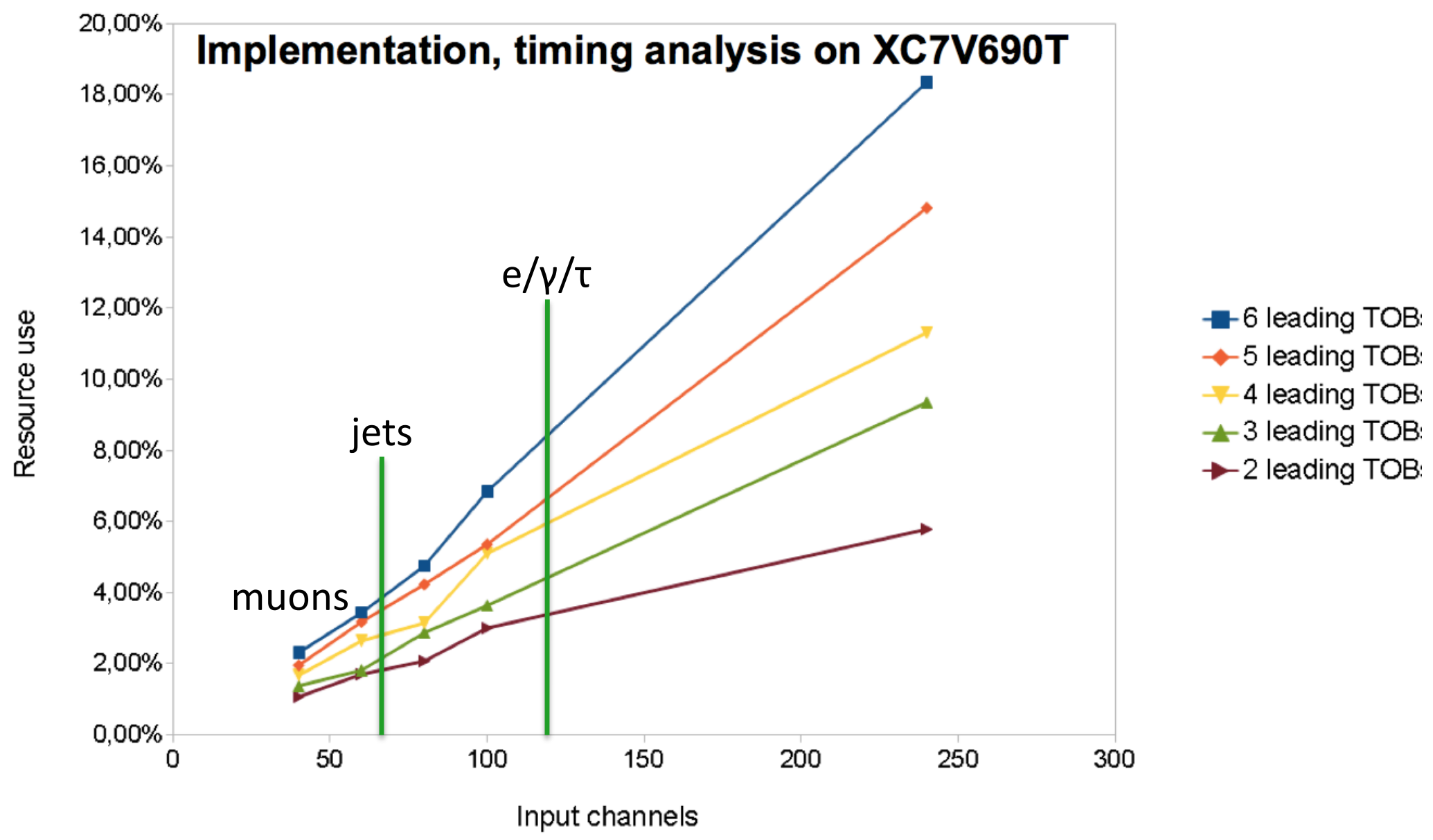




\section{Wrapping up...}

ATLAS trigger system

Future runs not sufficient to base decisions solely on $\mathrm{p}_{\mathrm{T}} / \mathrm{E}_{\mathrm{T}}$

Solution: Topological Trigger

future of trigger system

FPGA based algorithms

Prototype tests going well, soon full production

chips with more logic

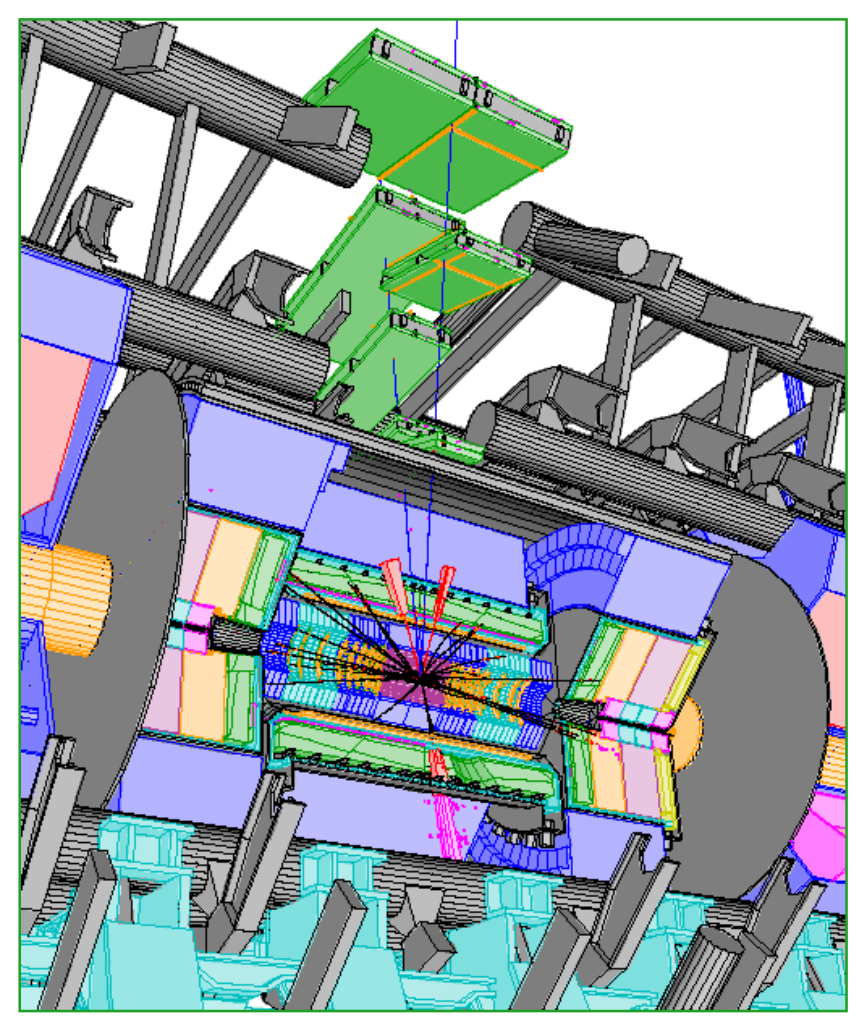

capacity, IO, and links 


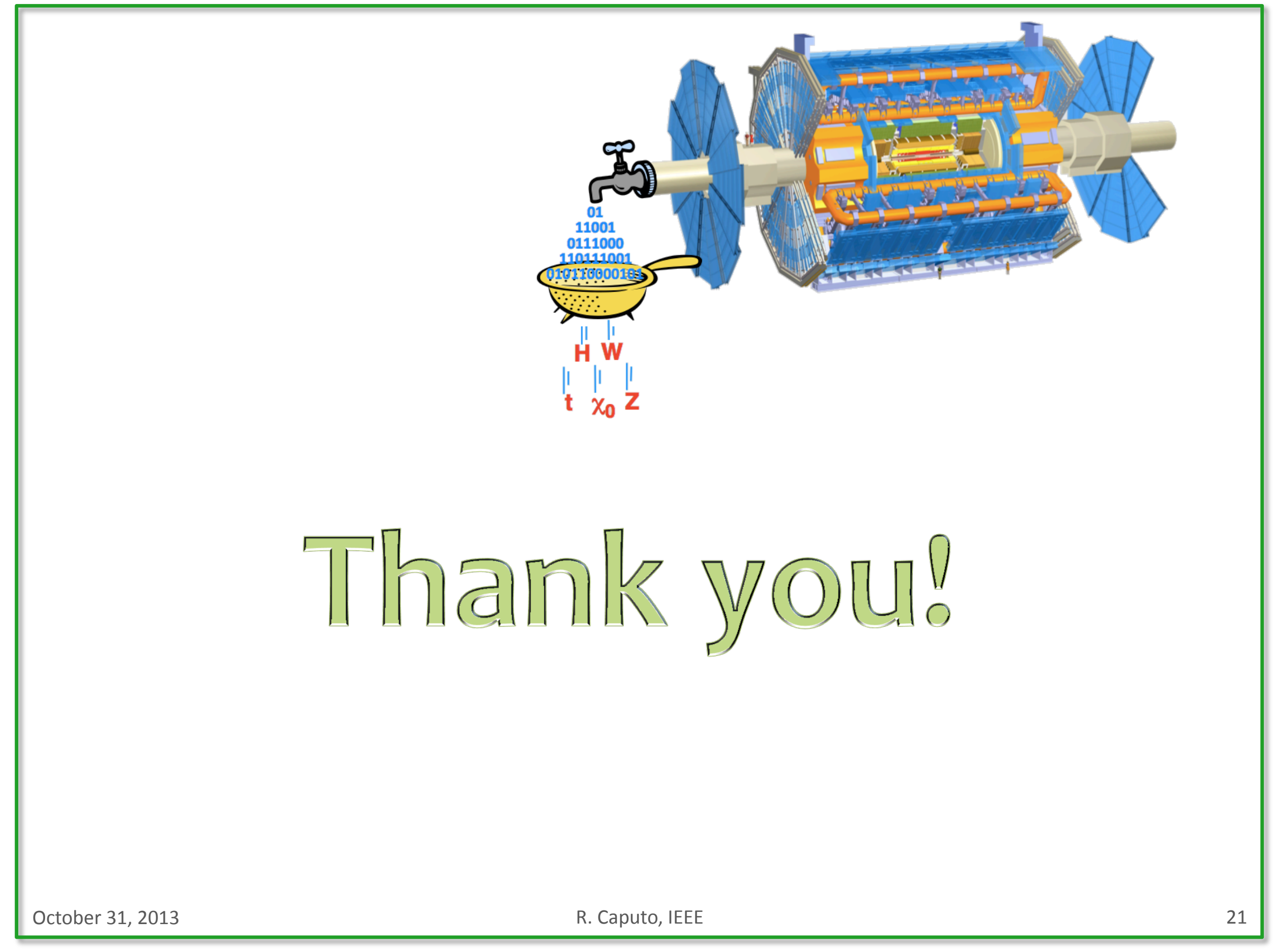

